\title{
CRUZAMENTOS DIALÉLICOS DE LINHAGENS DE MILHO SOB CONDIÇÕES DE MANCHA DE PHAEOSPHAERIA
}

\author{
Maria Elisa Ayres Guidetti Zagatto Paterniani2*; Eduardo Sawazaki; Christina Dudienas³; José \\ Carlos Vila Nova Alves Pereira ${ }^{4}$ \\ ${ }^{2}$ Centro de Plantas Graníferas - IAC, C.P. 28 - CEP: 13001-970 - Campinas, SP. \\ ${ }^{3}$ Centro de Fitossanidade - IAC. \\ ${ }^{4}$ Núcleo de Agronomia da Alta Mogiana - IAC, C.P. 271 - CEP: 14001-970 - Ribeirão Preto, SP. \\ *Autor correspondente <elisa@cec.iac.br>
}

RESUMO: Híbridos simples de milho resultantes de cruzamentos dialélicos entre dez linhagens endogâmicas provenientes do CIMMYT foram avaliados em 4 locais do Estado de São Paulo (Estações Experimentais do IAC), em 1996/97, quanto à estabilidade e adaptabilidade da produtividade de grãos. Os ensaios foram instalados sob delineamento de blocos casualizados com três repetições, incluindo quatro testemunhas comerciais. Foi verificada grande previsibilidade da produção e adaptabilidade ampla $(b=1,0)$ da maioria dos híbridos, indicando que o conjunto de linhagens é fonte potencial de híbridos heteróticos e de estabilidade satisfatória. Houve intensa severidade da mancha foliar de Phaeosphaeria maydis em Ribeirão Preto, considerada um dos fatores de estresse que mais contribuiu para a redução da qualidade ambiental. A doença foi avaliada cerca de 30 dias após o florescimento, através de uma escala de notas de 1 a 9 , correspondendo a $0 \%$ e $>80 \%$ de aréa foliar afetada na planta adulta, respectivamente. Houve uma tendência dos materiais mais resistentes à doença apresentarem valores de adaptabilidade menores que $1,0(b<1,0)$, sendo a correlação simples entre notas da doença e coeficientes de regressão $b(r=0,353)$ significativa pelo Teste $t$ a $2 \%$ de probabilidade. Os híbridos L04xL10 e L10xL11 tiveram as maiores produtividades do conjunto, não diferiram das testemunhas comerciais e demonstraram estabilidade de produção, sendo o último resistente à mancha de Phaeosphaeria.

Palavras-chave: Phaeosphaeria maydis, Zea mays, adaptabilidade, estabilidade, híbrido

\section{DIALLEL CROSSES AMONG MAIZE LINES UNDER PHAEOSPHAERIA LEAF SPOT INFESTATION}

\begin{abstract}
Single-cross hybrids of maize obtained from diallell crosses among ten inbred lines from CIMMYT were grown in four locations of the State of São Paulo, Brazil, during 1996/97, to evaluate stability and adaptability of grain yield. The experiments were set up in a randomized block design with three replications, including four comercial controls. High predictability of grain yield and general adaptability $(b=1,0)$ was observed for most hybrids, indicating that the lines are a potential source of heterosis as well as for good adaptability. A severe infestation of Phaeosphaeria maydis leaf spot occured in Ribeirão Preto, representing a stress factor responsible for reduction of environmental quality. The disease was assessed about 30 days after flowering, using a scale of grades of 1 to 9 , for $0 \%$ and $80 \%$ of the leaf area affected in the adult plant, respectively. There was a tendency of the more resistant materials to show adaptability values lower than $1.0(b<1.0)$, being the single correlation between disease evaluation and the regression coefficient $b(\mathrm{r}=0.353)$ significant at a 2\% $\mathrm{t}$ test. Hybrids L04XL10 and L10XL11 showed highest grain yield values, similar to the checks, and presenting stability also. It was noted that L10XL11 was resistant to Phaeosphaeria leaf spot.
\end{abstract}

Key words: Zea mays, Phaeosphaeria maydis, adaptability, stability, hybrids

\section{INTRODUÇÃO}

Linhagens de milho vigorosas, precoces e de dife-rentes procedências vêm sendo avaliadas no programa de melhoramento do IAC quanto à resistência às principais doenças foliares que vêm ocorrendo na cultura (Lima et al., 1996) e quanto à capacidade de combinação em cruzamentos dialélicos (Paterniani et al., 1998; Lima et al., 1998), visando à obtenção de híbridos heteróticos e com níveis satisfatórios de resistência genética.

Um dos problemas que vem ocasionando perdas na produtividade da cultura do milho é a incidência de doenças foliares, que vem ocorrendo de maneira intensa no Centro-Sul do Brasil. O aparecimento da ferrugem de Puccinia polysora no Estado de São Paulo, no início dos anos 90, provocou uma queda drástica da estabilidade de híbridos

${ }^{1}$ Trabalho apresentado na $18^{\text {a }}$ Reunion Latinoamericana del Maiz, Sete Lagoas, 1999. 
suscetíveis de elevada produtividade (Sawazaki, 1999¹). A crescente incidência e severidade da mancha foliar de Phaeosphaeria em milho vem reduzindo de forma drástica a produtividade e a estabilidade de materiais suscetíveis, sendo a identificação de genótipos resistentes fundamental dentro dos programas de melhoramento da cultura.Uma preocupação constante dos melhoristas de milho é a quantificação da interação genótipo $x$ ambiente, que fornece subsídios para a escolha de genótipos de adaptação ampla ou de adaptação restrita a ambientes específicos, atendendo a necessidades locais e regionais (Alves, 1996).

Análises de estabilidade e adaptabilidade permitem a identificação de genótipos de comportamento previsível e que sejam responsivos às variações do ambiente, em condições específicas ou amplas (Cruz \& Regazzi, 1994).

Adaptação e estabilidade, embora sejam fenômenos relacionados, não devem ser considerados como um só (Vencovsky \& Barriga, 1992). A estabilidade é considerada como a capacidade dos genótipos de exibirem um desempenho o mais constante possível, em função das variações da qualidade ambiental; o termo adaptabilidade designa a capacidade potencial dos genótipos para assimilarem vantajosamente o estímulo ambiental; uma vantagem do ponto de vista do rendimento agrícola (Mariotti et al., citado por Vencovsky \& Barriga, 1992).

Também existe uma corrente que prefere utilizar o termo adaptabilidade para designar adaptação a diferentes ambientes, como locais ou outras condições geográficas e estabilidade para se referir à habilidade dos genótipos de se adaptarem a flutuações climáticas ao longo de anos agrícolas, dentro de um dado local ou área geográfica. De fato, é esta última que interessa ao agricultor (Vencovsky \& Barriga, 1992).

Diversos métodos têm sido propostos para investigar a estabilidade (Eberhart \& Russell, 1966; Verma et al., 1978; Silva \& Barreto, 1985; Cruz et al., 1989; Lin \& Binns, 1992; Storck \& Vencovsky, 1994). A diferença entre os métodos origina-se nos próprios conceitos de estabilidade e nos procedimentos biométricos empregados para sua estimativa.

O método de Eberhart \& Russell (1966) baseiase na regressão linear, sendo cada genótipo caracterizado

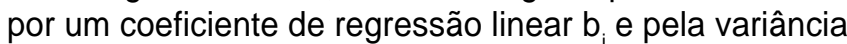
dos desvios de regressão $\left(\mathrm{s}^{2}{ }_{\mathrm{di}}\right)$, devendo ser o preferido quando o número de ambientes for inferior a sete (Vencovsky \& Barriga ,1992).

O presente trabalho tem por objetivo investigar a estabilidade e adaptabilidade de 45 híbridos simples experimentais de milho, obtidos a partir de um dialélico completo $10 \times 10$ de linhagens endogâmicas, avaliados em ambientes com características edafoclimáticas distintas do Estado de São Paulo, em condições de intensa severidade da mancha foliar de Phaeosphaeria maydis.

\section{MATERIAL E MÉTODOS}

Dez linhagens de milho provenientes do CIMMYT, denominadas L02, L03, L04, L05, L06, L08, L09, L10, L11 e $L 13$, derivadas respectivamente dos materiais MJ 2742, CML 109, CML110, CML 114, CML 115, CML 119, CML 124, CML 137, CML 138 e CML 153, com características agronômicas de porte e ciclo favoráveis, foram cruzadas em esquema dialélico completo. Os 45 híbridos resultantes foram avaliados na safra 1996/97, em quatro locais do Estado de São Paulo: Mococa (Estação de Agronomia/ $I A C)$, Campinas (Núcleo Experimental de Campinas/IAC), Ribeirão Preto (Núcleo de Agronomia da Alta Mogiana/IAC) e Assis (Núcleo de Agronomia do Vale do Paranapanema/ IAC), visando à obtenção das estimativas da capacidade geral e específica de combinação das linhagens em questão (Paterniani et al., 1998).

O delineamento experimental utilizado foi o de blocos ao acaso com três repetições e 49 tratamentos, sendo 45 híbridos experimentais e 4 testemunhas comerciais : AG 5011 (HT), C 333 (HS), P 3041 (HT) e Z 8392 (HS). As parcelas constituíram-se de duas linhas de $5 \mathrm{~m}$ de comprimento com espaçamento de $0,90 \mathrm{~m}$ entre linhas e 0,20 entre plantas.

A avaliação da incidência de Phaeosphaeria maydis foi efetuada cerca de 30 dias após o florescimento, utilizando-se uma escala de notas de 1 a 9 , respectivamente para $0 \%, 1 \%, 10 \%, 20 \%, 30 \%, 40 \%, 60 \%, 80 \%$ e $>$ de $80 \%$ de área foliar afetada, segundo o procedimento do Guia Agroceres de Sanidade. Foram avaliadas 5 plantas por parcela em Assis, Ribeirão Preto e Mococa; em Campinas a doença praticamente não ocorreu, predominando a ferrugem causada por Puccinia sorghi.

Foram efetuadas análises de variância individuais e conjuntas da produtividade e das notas de severidade (transformadas em $\sqrt{x}+1,0$ ).

Os parâmetros de estabilidade e adaptabilidade foram estimados com a utilização do programa Genes (Cruz, 1997), de acordo com o modelo de Eberhart \& Russell (1966), como se segue:

$Y_{i j}=m_{i}+b_{i j} l_{j}+d_{i j}+e_{i j}$, onde:

$Y_{\mathrm{ij}}$ : é a média do cultivar i no local $\mathbf{j}$;

$m_{i}$ : é o valor do caráter no cultivar $\mathbf{i}$ em condições ambientais médias;

$b_{i}$ : é o coeficiente de regressão linear; indica quanto o comportamento do cultivar i se altera com a alteração do ambiente $\left(\mathrm{I}_{\mathrm{j}}\right)$;

I: é o índice ambiental;

$\mathrm{d}_{\mathrm{ij}}$ : mede os desvios da regressão;

$\mathbf{e}_{\mathrm{ij}}$ : erro experimental contido em $\mathrm{Y}_{\mathrm{ij}}$

Obtiveram-se ainda coefícientes de regressão linear ( $r$ ) de acordo com Gomes (1990) entre o coeficiente de regressão (b) da análise da estabilidade e as notas de severidade de P.maydis em Ribeirão Preto e também entre notas de severidade de $P$. maydis e a produtividade de grãos em Ribeirão Preto. Foi considerado apenas este

'SAWAZAKI, E. (Instituto Agronômico, Campinas) Comunicação pessoal, 1999. 
local por ter havido maior severidade da doença aliada à queda de produtividade de grãos, constatando-se que o principal fator de estresse (menor qualidade ambiental) que interferiu na produtividade e estabilidade dos híbridos foi a doença em Ribeirão Preto.

\section{RESULTADOS E DISCUSSÃO}

A TABELA 1 mostra os valores médios da produtividade dos híbridos simples por local, o coeficiente de variação (C.V.\%) e a diferença mínima significativa pelo Teste

TABELA 1 - Produtividade média $\left(\mathrm{kg} \mathrm{ha}^{-1}\right)$ de 45 híbridos simples de milho resultantes de dialélico 10X10, em quatro locais do Estado de São Paulo. 1996/97.

\begin{tabular}{|c|c|c|c|c|c|c|}
\hline Tratamento & Hibrido & Mococa & Campinas & Ribeirão Preto & Assis & Média \\
\hline 1 & L02 X L03 & 7.247 & 7.273 & 4.591 & 4.705 & 5.954 \\
\hline 2 & L02 X L04 & 7.658 & 7.625 & 4.828 & 5.523 & 6.408 \\
\hline 3 & L02 X L05 & 7.118 & 7.513 & 5.518 & 7.185 & 6.834 \\
\hline 4 & L02 X L06 & 7.269 & 8.538 & 5.621 & 7.883 & 7.328 \\
\hline 5 & L02 X L08 & 6.773 & 6.723 & 3.397 & 5.947 & 5.710 \\
\hline 6 & L02 X L09 & 7.606 & 8.259 & 4.896 & 7.572 & 6.633 \\
\hline 7 & L02 X L10 & 8.830 & 9.408 & 5.756 & 6.868 & 7.716 \\
\hline 8 & L02 X L11 & 6.958 & 8.462 & 5.765 & 6.656 & 7.002 \\
\hline 9 & L02 X L13 & 7.923 & 7.984 & 6.591 & 5.730 & 7.057 \\
\hline 10 & L03 X L04 & 3.372 & 4.054 & 2.906 & 3.517 & 3.552 \\
\hline 11 & L03 X L05 & 7.248 & 6.758 & 5.141 & 5.577 & 6.178 \\
\hline 12 & L03 X L06 & 7.259 & 8.166 & 5.950 & 7.557 & 7.233 \\
\hline 13 & L03 X L08 & 7.985 & 7.940 & 6.035 & 7.102 & 7.266 \\
\hline 14 & L03 X L09 & 7.514 & 8.316 & 4.994 & 6.862 & 6.922 \\
\hline 15 & L03 X L10 & 8.811 & 9.928 & 6.542 & 7.646 & 8.232 \\
\hline 16 & L03 X L11 & 9.122 & 8.508 & 5.465 & 8.177 & 7.818 \\
\hline 17 & L03 X L13 & 8.532 & 8.848 & 5.827 & 6.979 & 7.546 \\
\hline 18 & L04 X L05 & 8.001 & 7.676 & 5.859 & 6.356 & 7.006 \\
\hline 19 & L04 X L06 & 7.541 & 8.844 & 6.064 & 8.211 & 7.665 \\
\hline 20 & L04 X L08 & 8.619 & 8.957 & 7.231 & 7.431 & 8.060 \\
\hline 21 & L04 X L09 & 8.494 & 8.673 & 5.645 & 7.933 & 7.686 \\
\hline 22 & L04 X L10 & 9.290 & 10.375 & 7.326 & 9.726 & 8.992 \\
\hline 23 & L04 X L11 & 8.743 & 9.380 & 5.276 & 7.737 & 7.951 \\
\hline 24 & L04 X L13 & 8.269 & 8.575 & 5.999 & 6.922 & 7.441 \\
\hline 25 & L05 X L06 & 4.543 & 6.042 & 4.170 & 6.504 & 5.315 \\
\hline 26 & L05 X L08 & 7.713 & 7.416 & 6.175 & 5.879 & 6.796 \\
\hline 27 & L05 X L09 & 8.081 & 8.638 & 5.954 & 7.360 & 7.508 \\
\hline 28 & L05 X L10 & 8.123 & 8.372 & 5.775 & 7.523 & 7.448 \\
\hline 29 & L05 X L11 & 8.060 & 7.790 & 5.925 & 7.901 & 7.419 \\
\hline 30 & L05 X L13 & 7.390 & 8.658 & 5.684 & 7.413 & 7.286 \\
\hline 31 & L06 X L08 & 7.200 & 7.745 & 5.678 & 6.611 & 6.808 \\
\hline 32 & L06 X L09 & 7.694 & 8.367 & 6.345 & 7.489 & 7.474 \\
\hline 33 & L06 X L10 & 8.670 & 9.183 & 7.263 & 8.286 & 8.350 \\
\hline 34 & L06 X L11 & 7.931 & 8.287 & 6.544 & 8.195 & 7.734 \\
\hline 35 & L06 X L13 & 8.051 & 8.710 & 6.776 & 7.499 & 7.759 \\
\hline 36 & L08 X L09 & 8.129 & 8.454 & 5.093 & 6.857 & 7.133 \\
\hline 37 & L08 X L10 & 7.987 & 9.555 & 6.842 & 8.744 & 8.282 \\
\hline 38 & L08 X L11 & 7.318 & 8.191 & 5.531 & 6.429 & 6.867 \\
\hline 39 & L08 X L13 & 8.094 & 8.131 & 6.076 & 7.178 & 7.370 \\
\hline 40 & L09 X L10 & 5.739 & 6.785 & 4.080 & 5.012 & 5.404 \\
\hline 41 & L09 X L11 & 8.336 & 9.595 & 6.125 & 8.958 & 8.254 \\
\hline 42 & L09 X L13 & 8.390 & 8.898 & 6.669 & 8.056 & 8.003 \\
\hline 43 & L10 X L11 & 9.362 & 10.208 & 7.317 & 8.709 & 8.899 \\
\hline 44 & L10 X L13 & 8.665 & 9.166 & 7.296 & 7.651 & 8.253 \\
\hline 45 & L11 X L13 & 8.296 & 9.031 & 6.885 & 7.565 & 7.944 \\
\hline 46 & AG 5011 & 9.053 & 10.301 & 8.635 & 10.558 & 9.637 \\
\hline 47 & C 333 & 9.105 & 9.984 & 8.190 & 9.621 & 9.225 \\
\hline 48 & P 3041 & 9.796 & 10.284 & 8.587 & 10.362 & 9.757 \\
\hline 49 & Z 8392 & 8.321 & 9.669 & 8.074 & 9.402 & 8.866 \\
\hline Média & & 7.890 & 8.454 & 6.023 & 7.346 & 7.428 \\
\hline C.V.\% & & 8,1 & 6,9 & 10,6 & 12,3 & 9,3 \\
\hline \multicolumn{2}{|c|}{ dms (Tukey $5 \%$ ) } & 2.144 & 1.951 & 2.128 & 3.010 & 1.122 \\
\hline
\end{tabular}


de Tukey. Elevados valores são observados em Campinas e Mococa, onde alguns híbridos simples tiveram produtividade compatível com a das testemunhas comerciais.

A mancha de $P$. maydis ocorreu com baixa severidade em Assis, sendo as notas médias dos híbridos simples experimentais e das testemunhas iguais a 2,5 e 2,2 , respectivamente; não houve portanto discriminação dos híbridos quanto à resistência à doença neste local, onde predominou a ferrugem de Puccinia polysora. Em Mococa a mancha de Phaeosphaeria ocorreu com severidade alta mas tardiamente, no final do ciclo do milho, não afetando substancialmente a produtividade de grãos. As médias de doença nos híbridos experimentais e nas testemunhas foram de 4,8 e 3,7, respectivamente. Em Ribeirão Preto houve maior amplitude de variação de notas de $P$. maydis, maior severidade da doença e redução drástica da produtividade de grãos.

Os resultados da análise da variância conjunta resumida são apresentados na TABELA 2 e indicam diferenças significativas a $1 \%$ de probabilidade entre ambientes $(A)$, genótipos $(G)$ e $A / G(G \times A+A)$, confirmando a enorme diversidade entre híbridos e locais. Foi ainda detectada a interação genótipo por ambiente (GXA), premissa básica para se proceder à análise de estabilidade.

A presença de interação $G \times A$ é ponto crítico nas pesquisas de melhoramento de plantas, influenciando o ganho de seleção e dificultando a indicação de cultivares de ampla adaptabilidade. Grandes mudanças na colocação dos genótipos em diferentes locais sugerem o desenvolvimento de cultivares específicos para diferentes locais ou a subdivisão de áreas geográficas (Duarte \& Paterniani, 1998). A identificação de cultivares com estabilidade de produção é outra alternativa para atenuar os efeitos da interação.

$\mathrm{Na}$ TABELA 3 apresentam-se as estimativas dos parâmetros de estabilidade e adaptabilidade de acordo com Eberhart \& Russell (1966), revelando que de maneira geral o conjunto de linhagens em questão originou híbridos

TABELA 2 - Análise da variância conjunta resumida da produtividade de grãos $\left(\mathrm{kg} \mathrm{ha}^{-1}\right)$ de 49 híbridos simples de milho, em quatro ambientes do Estado de São Paulo (1996/97).

\begin{tabular}{lrrr}
\hline Causa da variação & G.L. & \multicolumn{1}{c}{ Q.M. } & \multicolumn{1}{c}{ F } \\
\hline Ambiente (A) & 3 & 159.050 .080 & $33,2^{* *}$ \\
Genótipo (G) & 48 & 14.996 .203 & $31,3^{\star *}$ \\
G x A & 144 & 882.830 & $1,8^{\star *}$ \\
A/G & 147 & 4.110 .733 & $8,6^{\star *}$ \\
\hline Resíduo médio & 384 & 478.569 & \\
\hline
\end{tabular}

${ }^{* *}$ significativo a $1 \%$. de estabilidade satisfatória (desvios da regressão não significativos). Incluem-se ainda as notas médias de P. maydis em Ribeirão Preto, onde a doença ocorreu com maior severidade e provocou queda da produtividade de grãos.

Dos 45 híbridos experimentais avaliados, 39 tiveram adaptabilidade ampla ou geral $(b=1,0)$, quatro demostraram adaptabilidade específica para ambientes favoráveis $(b>1,0)$ e um para ambientes desfavoráveis $(b<1,0)$.

Apresentaram problemas de estabilidade (desvios significativos) os cruzamentos: L2xL3, L02xL04, L02xL13, L03xL11, L05xL06, L05xL08, L09xL11, bem como os híbridos comerciais AG 5011 e Z 8392. Verificaram-se condições adversas representadas pela alta severidade da mancha de Phaeosphaeria maydis em Ribeirão Preto, o que explica os valores de $b<1,0$ nas testemunhas comerciais, que têm maior resistência genética à doença.

Destacaram-se pela resistência a $P$. maydis (notas $\cong 3,0):$ L02XL05, L02XL06, L02XL08, L03XL05, L04XL05, L04XL06, L05XL08, L05XL09, L05XL10, L05XL11, L05XL13 e L06XL08, evidenciando-se a L05 como fonte potencial de resistência à doença.

Com relação aos coeficientes de regressão simples, foram obtidos valores de $r=0,353$ entre a adaptabilidade dos híbridos (b) obtida da análise da estabilidade e notas de $P$. maydis; entre notas da doença e produtividade de cada material em Ribeirão Preto, o valor de $r$ calculado foi de -0,340; ambos os valores foram significativos a $2 \%$ pelo Teste $t$ (TABELA 3 ).

Os híbridos L03xL04, L05xL06 e L09xL10 tiveram o pior desempenho, provavelmente devido à pequena divergência genética entre as linhagens progenitoras e à sua alta suscetibilidade a doenças.

Os híbridos de maior produtividade (L04XL10 e L10xL11) apresentaram adaptabilidade geral $(b=1,0)$ e boa previsibilidade de produção de grãos (variância dos desvios nula), o que fornece subsídios para o lançamento comercial a curto-médio prazo. Estas linhagens destacaram-se com estimativas de capacidade geral de combinação positivas para peso de grãos (Paterniani et al., 1998).

Os elevados valores do coeficiente de determinação $\left(R^{2}\right)$ indicaram que os cultivares se adequaram ao modelo de estabilidade utilizado.

Os híbridos das linhagens L02 e L11 tiveram em geral $b \geq 1,0$, o que indica certo controle genético da estabilidade. Constatou-se ainda que os híbridos da L10 foram estáveis e tiveram elevada produtividade média. Vencovsky \& Torres, citados por Vencovsky \& Barriga (1992), obtiveram indicações de que o padrão de resposta dos cultivares quanto à estabilidade e adaptabilidade tem um grau de controle genético que pode ser explorado pelo melhoramento.

Os resultados ressaltaram a possibilidade de se associar elevada produtividade de grãos com adaptabilidade geral e estabilidade em híbridos específicos das linhagens em questão. 
TABELA 3 - Produtividade média, notas de severidade de Phaeosphaeria maydis, estimativas de parâmetros de estabilidade pelo método de Eberhart \& Russel (1966) e coeficientes de determinação $\left(R^{2}\right)$ de 45 híbridos simples de milho resultantes de dialélico 10X10, em quatro locais do Estado de São Paulo. 1996/97.

\begin{tabular}{|c|c|c|c|c|c|c|c|}
\hline Trat. & Híbrido & Média & Média (R. Preto) $)^{(4)}$ & P. maydis ${ }^{(3)}$ & $b_{i}^{(1)}$ & $\mathrm{s}^{2} \mathrm{~d}_{\mathrm{i}}^{(2)}$ & $\mathrm{R}^{2}$ \\
\hline & & $\mathrm{kg} \mathrm{ha}^{-1}$ & $\mathrm{~kg} \mathrm{ha}^{-1}$ & 1 a 9 & & $\mathrm{~kg} \mathrm{ha}^{-1}$ & \\
\hline 1 & L02 X L03 & 5.954 & 4.591 & 6,7 & 1,22 & $831577^{\star \star}$ & 0,71 \\
\hline 2 & L02 X L04 & 6.408 & 4.828 & 5,1 & 1,27 & $390746^{*}$ & 0,83 \\
\hline 3 & L02 X L05 & 6.834 & 5.518 & 2,8 & 0,82 & -41251 & 0,90 \\
\hline 4 & L02 X L06 & 7.328 & 5.621 & 3,0 & 1,10 & 225205 & 0,84 \\
\hline 5 & L02 X L08 & 5.710 & 3.397 & 3,2 & $1,47^{\star}$ & 131048 & 0,92 \\
\hline 6 & L02 X L09 & 6.633 & 4.896 & 6,5 & $1,43^{*}$ & 216291 & 0,90 \\
\hline 7 & L02 X L10 & 7.716 & 5.756 & 4,3 & $1,56^{\star *}$ & 209019 & 0,92 \\
\hline 8 & L02 X L11 & 7.002 & 5.765 & 5,1 & 0,99 & 81519 & 0,87 \\
\hline 9 & L02 X L13 & 7.057 & 6.591 & 4,7 & 0,65 & $946146^{* *}$ & 0,42 \\
\hline 10 & L03 X L04 & 3.552 & 2.906 & 6,8 & $0,46^{*}$ & -158580 & 0,99 \\
\hline 11 & L03 X L05 & 6.178 & 5.141 & 2,6 & 0,80 & 265199 & 0,71 \\
\hline 12 & L03 X L06 & 7.233 & 5.950 & 4,0 & 0,85 & -9325 & 0,88 \\
\hline 13 & L03 X L08 & 7.266 & 6.035 & 6.6 & 0,85 & -81446 & 0.94 \\
\hline 14 & L03 X L09 & 6.922 & 4.994 & 7.1 & 1.36 & -157461 & 0.99 \\
\hline 15 & L03 X L10 & 8.232 & 6.542 & 5,8 & 1,36 & 24244 & 0,94 \\
\hline 16 & L03 X L11 & 7.818 & 5.465 & 5,0 & 1,41 & $521138^{*}$ & 0,83 \\
\hline 17 & L03 X L13 & 7.546 & 5.827 & 6,0 & 1,31 & 27380 & 0,94 \\
\hline 18 & L04 X L05 & 7.006 & 5.859 & 2,6 & 0,86 & 143546 & 0,80 \\
\hline 19 & L04 X L06 & 7.665 & 6.064 & 2,9 & 1,03 & 237821 & 0,81 \\
\hline 20 & L04 X L08 & 8.060 & 7.231 & 6,2 & 0,74 & 57545 & 0,80 \\
\hline 21 & L04 X L09 & 7.686 & 5.645 & 5,8 & 1,30 & 7330 & 0,94 \\
\hline 22 & L04 X L10 & 8.992 & 7.326 & 5,1 & 1,20 & -111684 & 0,98 \\
\hline 23 & L04 X L11 & 7.951 & 5.276 & 4,8 & $1,71^{\star *}$ & 10576 & 0,95 \\
\hline 24 & L04 X L13 & 7.441 & 5.999 & 6,0 & 1,11 & -10963 & 0,93 \\
\hline 25 & L05 X L06 & 5.315 & 4.170 & 4,0 & 0,58 & $1208277^{\star \star}$ & 0,29 \\
\hline 26 & L05 X L08 & 6.796 & 6.175 & 2,6 & 0,62 & $446251^{*}$ & 0,50 \\
\hline 27 & L05 X L09 & 7.508 & 5.954 & 3,3 & 1,11 & -156024 & 1,00 \\
\hline 28 & L05 X L10 & 7.448 & 5.775 & 2,9 & 1,11 & -103180 & 0,97 \\
\hline 29 & L05 X L11 & 7.419 & 5.925 & 2,6 & 0,84 & 193583 & 0,76 \\
\hline 30 & L05 X L13 & 7.286 & 5.684 & 3,2 & 1,14 & -25683 & 0,94 \\
\hline 31 & L06 X L08 & 6.808 & 5.678 & 3,0 & 0,85 & -147202 & 0,99 \\
\hline 32 & L06 X L09 & 7.474 & 6.345 & 4,0 & 0,80 & -142538 & 0,98 \\
\hline 33 & L06 X L10 & 8.350 & 7.263 & 3,3 & 0,78 & -158149 & 0,99 \\
\hline 34 & L06 X L11 & 7.734 & 6.544 & 3,2 & 0,71 & 21697 & 0,82 \\
\hline 35 & L06 X L13 & 7.759 & 6.776 & 3,5 & 0,77 & -120195 & 0,96 \\
\hline 36 & L08 X L09 & 7.133 & 5.093 & 6,0 & $1,45^{\star}$ & -79871 & 0,97 \\
\hline 37 & L08 X L10 & 8.282 & 6.842 & 3,7 & 0,97 & 304279 & 0,77 \\
\hline 38 & L08 X L11 & 6.867 & 5.531 & 4,0 & 1,07 & -57402 & 0,95 \\
\hline 39 & L08 X L13 & 7.370 & 6.076 & 4,5 & 0,90 & -91411 & 0,95 \\
\hline 40 & L09 X L10 & 5.404 & 4.080 & 4,8 & 1,07 & -44177 & 0,94 \\
\hline 41 & L09 X L11 & 8.254 & 6.125 & 4,3 & 1,34 & $345894^{*}$ & 0,85 \\
\hline 42 & L09 X L13 & 8.003 & 6.669 & 6,6 & 0,91 & -148655 & 0,99 \\
\hline 43 & L10 X L11 & 8.899 & 7.317 & 3,2 & 1,17 & -144224 & 0,99 \\
\hline 44 & L10 X L13 & 8.253 & 7.296 & 4,0 & 0,68 & 44644 & 0,78 \\
\hline 45 & L11 X L13 & 7.944 & 6.885 & 3,6 & 0,86 & -78647 & 0,94 \\
\hline 46 & AG 5011 & 9.637 & 8.635 & 2,0 & $0,54^{*}$ & $688882^{* *}$ & 0,36 \\
\hline 47 & C 333 & 9.225 & 8.190 & 2,5 & 0,66 & 40939 & 0,78 \\
\hline 48 & P 3041 & 9.757 & 8.587 & 2,7 & 0,66 & 133413 & 0,71 \\
\hline 49 & Z 8392 & 8.866 & 8.074 & 4,6 & $0,51^{*}$ & $353752^{*}$ & 0,45 \\
\hline
\end{tabular}

${ }^{(1)}$ Coeficiente de regressão linear; * $e^{* *}$ : significativamente diferente de um a 5 e $1 \%$ pelo teste $\mathrm{t}$;

(2) Variância dos desvios de regressão; * $\mathrm{eC}{ }^{* *}$ : significativa a 5 e $1 \%$ pelo teste $F .(3)$ notas médias em Ribeirão Preto. $r_{13}=0,353$ e $r_{3,4}=-0,340$ (significativos a $2 \%$ pelo Teste t). 


\section{CONCLUSÕES}

- Híbridos de elevada produtividade e previsibilidade podem ser obtidos a partir do conjunto de linhagens utilizado.

- Os híbridos mais produtivos (L04XL10 e L10xL11) tiveram adaptabilidade geral $(b=1,0)$ e previsibilidade (estabilidade), destacando-se por não diferirem significativamente das testemunhas comerciais.

- A mancha de Phaeosphaeria maydis influenciou na adaptabilidade dos híbridos de milho.

\section{AGRADECIMENTO}

Os autores agradecem à FAPESP pelo financiamento do projeto.

\section{REFERÊNCIAS BIBLIOGRÁFICAS}

ALVES, S.J. Estudo dos efeitos ambientais, estabilidade e adaptabilidade genética em milho. Londrina, 1996. 129p. Dissertação (Mestrado) - Universidade Estadual de Londrina.

CRUZ, C.D. Programa Genes: aplicativo computacional em genética e estatística. Viçosa: UFV, 1997. 442p.

CRUZ, C.D.; REGAZZI, A.J. Modelos biométricos aplicados ao melhoramento genético. Viçosa: UFV, 1994. 390p.

CRUZ, C.D.; TORRES, R.A.; VENCOVSKY, R. An alternative approach to the stability analysis proposed by Silva and Barreto. Revista Brasileira de Genética, v.12, p.567-580, 1989.

DUARTE, A.P.; PATERNIANI, M.E.A.G.Z. (Coord.) Cultivares de milho no Estado de São Paulo. Campinas: Instituto Agronômico, 1998. 81p. (Documentos, 62)

EBERHART , S.A.; RUSSELL, W.A. Stability parameters for comparing varieties. Crop Science, v.6, p.36-40, 1966.
GOMES, F.P. Curso de estatística experimental. 13.ed. Piracicaba: Nobel, 1990.467p.

LIMA, M.; PATERNIANI, M.E.A.G.Z.; DUDIENAS, C.; SIQUEIRA, W.J.; SAWAZAKI, E.; SORDI, G. DE. Avaliação da resistência à ferrugem tropical em linhagens de milho. Bragantia, v.55, p.269-273, 1996.

LIMA, M.; DUDIENAS, C.; PATERNIANI, M.E.A.G.Z.; GALLO, P.B. Cruzamentos parciais entre linhagens de milho com ênfase na produtividade e doenças foliares. In: CONGRESSO NACIONAL DE MILHO E SORGO, 22., Recife, 1998. Resumos. Recife: IPA, 1998. p.38.

LIN, C.S.; BINNS, M.R. Genetic properties of four types of stability parameter. Theoretical and Applied Genetics, v.82, p.505-509, 1992.

PATERNIANI, M.E.A.G.Z.; SAWAZAKI, E.; DUDIENAS, C.; DUARTE, A.P.; GALLO, P.B. Cruzamentos dialélicos entre linhagens de milho com diferentes níveis de resistência a doenças foliares. In: CONGRESSO NACIONAL DE MILHO E SORGO, 22., Recife, 1998. Resumos. Recife: IPA, 1998 p.98.

SILVA, J.G.; BARRETO, J.N. Aplicação da regressão linear segmentada em estudos da interação genótipo por ambiente. In: SIMPÓSIO DE ESTATÍSTICA APLICADA À EXPERIMENTAÇÃO AGRONÔMICA, 1., Piracicaba,1985. Campinas: Fundação Cargill, 1985. p.49-50.

STORCK, L.; VENCOVSKY, R. Stability analysis based on a bisegmented discontinouos model with measurement errors in the variables. Revista Brasileira de Genética, v.17, p.75-81, 1994.

VENCOVSKY, R.; BARRIGA, P. Genética biométrica no fitomelhoramento. Ribeirão Preto: Sociedade Brasileira de Genética, 1992. 496p.

VERMA, M.M.; CHAHAL, G.S.; MURTY, B.R. Limitations of conventional regression analysis: a proposed modification. Theoretical and Applied Genetics, v.53, p.89-91, 1978.

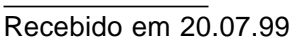

